\title{
Studies of quantum-mechanical coherency effects in neutrino-nucleus elastic scattering
}

\author{
V. Sharma, ${ }^{1,2}$ L. Singh, ${ }^{1,3}$ H. T. Wong $\odot{ }^{1,{ }^{*}}$ M. Agartioglu, ${ }^{1,4,5}$ J.-W. Chen, ${ }^{6}$ M. Deniz, ${ }^{4}$ S. Kerman, ${ }^{4, \dagger}$ H. B. Li, ${ }^{1}$ \\ C.-P. Liu, ${ }^{5}$ K. Saraswat, ${ }^{1}$ M. K. Singh, ${ }^{1,2}$ and V. Singh ${ }^{2,3}$ \\ (TEXONO Collaboration) \\ ${ }^{1}$ Institute of Physics, Academia Sinica, Taipei 11529, Taiwan \\ ${ }^{2}$ Department of Physics, Institute of Science, Banaras Hindu University, Varanasi 221005, India \\ ${ }^{3}$ Department of Physics, School of Physical and Chemical Sciences, Central University of South Bihar, \\ Gaya 824236, India \\ ${ }^{4}$ Department of Physics, Dokuz Eylül University, Buca, İzmir 35160, Turkey \\ ${ }^{5}$ Department of Physics, National Dong Hwa University, Shoufeng, Hualien 97401, Taiwan \\ ${ }^{6}$ Department of Physics, CTS and LeCosPA, National Taiwan University, Taipei 10617, Taiwan
}

(Received 14 October 2020; revised 1 March 2021; accepted 12 April 2021; published 7 May 2021)

\begin{abstract}
Neutrino-nucleus elastic scattering $\left(\nu \mathrm{A}_{e l}\right)$ provides a unique laboratory to study the quantum-mechanical (QM) coherency effects in electroweak interactions. The deviations of the cross sections from those of completely coherent systems can be quantitatively characterized through a coherency parameter $\alpha\left(q^{2}\right)$. The relations between $\alpha$ and the underlying nuclear physics in terms of nuclear form factors are derived. The dependence of cross section on $\alpha\left(q^{2}\right)$ for the various neutrino sources is presented. The $\alpha\left(q^{2}\right)$ values are evaluated from the measured data of the COHERENT CsI and Ar experiments. Complete coherency and decoherency conditions are excluded by the CsI data with $p=0.004$ at $q^{2}=3.1 \times 10^{3} \mathrm{MeV}^{2}$ and $p=0.016$ at $q^{2}=2.3 \times 10^{3} \mathrm{MeV}^{2}$, respectively, verifying that both $\mathrm{QM}$ superpositions and nuclear many-body effects contribute to $\nu \mathrm{A}_{e l}$ interactions.
\end{abstract}

DOI: 10.1103/PhysRevD.103.092002

\section{INTRODUCTION}

The elastic scattering of a neutrino with a nucleus [1,2]

$$
\nu \mathrm{A}_{e l}: \quad \nu+A(Z, N) \rightarrow \nu+A(Z, N)
$$

where $A(Z, N)$ denotes the atomic nucleus with its respective atomic, charge, and neutron numbers, is a fundamental electroweak neutral current process in the Standard Model (SM).

Studies of neutrino-nucleus elastic scattering can provide sensitive probes to physics beyond the SM (BSM) $[3,4]$ and certain astrophysical processes [1,5]. It offers prospects to study quantum-mechanical (QM) coherency effects in

\footnotetext{
* Corresponding author. htwong@phys.sinica.edu.tw

Deceased.

Published by the American Physical Society under the terms of the Creative Commons Attribution 4.0 International license. Further distribution of this work must maintain attribution to the author(s) and the published article's title, journal citation, and DOI. Funded by SCOAP .
}

electroweak interactions [6] and neutron density distributions [7], to detect supernova neutrinos [8], and to provide a compact and transportable neutrino detectors for real-time monitoring of nuclear reactors [9]. The $\nu \mathrm{A}_{e l}$ events from solar and atmospheric neutrinos are the irreducible "neutrino floor" background [10] to the coming generations of dark matter experiments [11].

There are several active experimental programs to observe and measure the $\nu \mathrm{A}_{e l}$ processes with neutrinos from reactors [12] or from decay-at-rest pions $(\mathrm{DAR}-\pi)$ [4] provided by a spallation neutron source [13]. Future dark matter experiments may also be sensitive to $\nu \mathrm{A}_{e l}$ from solar neutrinos [14]. First positive measurement of $\nu \mathrm{A}_{e l}$ was achieved by the COHERENT experiment with $\mathrm{CsI}(\mathrm{Na})$ detector [15], followed by measurements with a liquid $\mathrm{Ar}$ detector [16].

The $\nu \mathrm{A}_{e l}$ interaction provides a laboratory to probe the QM coherency effects [6]. Experimental measurements are mostly performed in a parameter space where the coherency effects are partial and incomplete. The deviations from perfect coherency would have to be described and quantified before this interaction can be effectively applied toward other goals like the studies of BSM physics. 
Coherency in QM superpositions among scattering amplitudes from individual nucleons is central to $\nu \mathrm{A}_{e l}$ interactions. Our earlier work [6] identified a coherency parameter $\alpha\left(q^{2}\right)$ which can quantify and consistently characterize the degree of coherency in $\nu \mathrm{A}_{e l}$ with different $\nu$ sources and target nuclei. This article follows and expands on these studies. The relations between $\alpha\left(q^{2}\right)$ with the complementary descriptions in terms of nuclear physics via the language of nuclear form factors or with the reduction in cross sections are discussed in Sec. II. The dependence of coherency effects with interaction kinematics for various neutrino sources and detector targets are surveyed in Sec. III. The constraints provided by the COHERENT CsI and Ar data are derived in Sec. IV.

\section{FORMULATION AND CHARACTERIZATION}

The $\nu \mathrm{A}_{e l}$ differential cross section at three-momentum transfer $q(\equiv|\vec{q}|)$ and neutrino energy $E_{\nu}$ can be expressed as $[2,6]$

$$
\left[\frac{d \sigma}{d q^{2}}\left(q^{2}, E_{\nu}\right)\right]_{\nu \mathrm{A}_{e l}}=\frac{1}{2}\left[\frac{G_{F}^{2}}{4 \pi}\right] \cdot\left[1-\frac{q^{2}}{4 E_{\nu}{ }^{2}}\right] \cdot \Gamma\left(q^{2}\right),
$$

where $\Gamma\left(q^{2}\right)$ is a function describing the contributions due to many-body physics in the target nuclei since the $\nu \mathrm{A}_{e l}$ interactions involve collective contributions of individual nucleons in the nucleus.

The relevant kinematics variable is $q^{2}$, which characterizes the physics and is universal to all targets. The experimental observable is the nuclear recoil energy $(T)$, expressed in units of $\mathrm{keV}_{\mathrm{nr}}$ in this article, which depends on the target nuclear mass $M$ and is related to $q^{2}$ via $q^{2}=2 M T+T^{2} \simeq 2 M T$. The minimal observable energy $T_{\min }$ for the nuclear recoils is the detector threshold, while kinematics limits the maximum recoil energy to $T_{\max }=$ $2 E_{\nu}{ }^{2} /\left(M+2 E_{\nu}\right) \simeq 2 E_{\nu}{ }^{2} / M$. These limits can be translated to $q_{\min }^{2}=2 M T_{\min }$ and $q_{\max }^{2}=4 E_{\nu}{ }^{2}\left[M /\left(M+2 E_{\nu}\right)\right] \simeq 4 E_{\nu}{ }^{2}$. The variations of the $\nu \mathrm{A}_{e l}$ differential and integral cross sections with respect to $T$ are discussed in the Appendix.

Depending on the particular physics aspects to probe, there are complementary formulations on the $\Gamma\left(q^{2}\right)$ function. The conventional description is based on nuclear physics, expressed as

$$
\Gamma\left(q^{2}\right) \equiv \Gamma_{N P}\left(q^{2}\right)=\left[\varepsilon Z F_{Z}\left(q^{2}\right)-N F_{N}\left(q^{2}\right)\right]^{2},
$$

where $F_{Z}\left(q^{2}\right) \in[0,1]$ and $F_{N}\left(q^{2}\right) \in[0,1]$ are, respectively, the proton and neutron nuclear form factors for the nucleus $A(Z, N)$, while $\varepsilon \equiv\left(1-4 \sin ^{2} \theta_{W}\right)=0.045$, indicating that the dominant contributions are from the neutrons.

The merit of this description is to connect $\nu \mathrm{A}_{e l}$ to nuclear physics so that its studies may benefit from or contribute to the wealth of information and data. Electron-nucleus scattering experiments provide important data to the nuclear proton form factor $F_{Z}\left(q^{2}\right)$ [17]. The neutron counterpart $F_{N}\left(q^{2}\right)$, however, would require weak processes to probe. Studies of $\nu \mathrm{A}_{e l}$ have therefore triggered intense activities toward their measurements [7], complementing experiments with parity-violation scattering using polarized electrons [18].

In the kinematics regime relevant to this work, $q^{2} R^{2} \ll$ $\pi^{2}$ (natural units with $\hbar=c=1$ are used throughout), where $R=1.2 A^{1 / 3} \mathrm{fm}$ is the typical scale characterizing the radius of nuclei, nucleons can be taken as structureless pointlike particles such that their internal dynamics and QCD effects can be neglected. At $q^{2} \rightarrow 0$, there is a perfect alignment of the scattering amplitude vectors of individual nucleons in the target nucleus [6]. The interactions are completely coherent. As $q^{2}$ increases, deviation from this complete coherency condition leads to a reduction in the cross section. The degree of coherency can be quantified by a parameter $\alpha\left(q^{2}\right) \equiv \cos \phi \in[0,1]$, where $\phi\left(q^{2}\right) \in[0, \pi / 2]$ is the misalignment phase angle between the scattering amplitudes of two nonidentical nucleons [6]. This leads to a formulation in terms of $\mathrm{QM}$ superpositions among the various scattering centers in which

$$
\begin{aligned}
\Gamma\left(q^{2}\right) & \equiv \Gamma_{Q M}\left(q^{2}\right) \\
& =Z \varepsilon^{2}[1+\alpha(Z-1)]+N[1+\alpha(N-1)]-2 \alpha \varepsilon Z N \\
& =(\varepsilon Z-N)^{2} \cdot \alpha\left(q^{2}\right)+\left(\varepsilon^{2} Z+N\right) \cdot\left[1-\alpha\left(q^{2}\right)\right] .
\end{aligned}
$$

The $\Gamma_{Q M}$ formulation with $\alpha\left(q^{2}\right)$ provides an intuitive physics understanding and quantitative description on the reduction of $\nu \mathrm{A}_{e l}$ cross sections in terms of QM phaseangle alignment and coherency. In particular, it naturally leads to the limiting behavior at the complete coherency $\left(\alpha=1\right.$ at $\left.q^{2} \sim 0\right)$ and complete decoherency $(\alpha=0$ at $\left.q^{2} \gtrsim[\pi / R]^{2}\right)$ states, corresponding to $\left(d \sigma / d q^{2}\right) \propto[\varepsilon Z-N]^{2}$ and $\left(d \sigma / d q^{2}\right) \propto\left[\varepsilon^{2} Z+N\right]$, respectively. The experimentally measured $\alpha\left(q^{2}\right)$ values from different isotope targets can be directly compared to reveal their varying degrees of coherency in the respective processes.

An alternative measurement-driven description, denoted by $\xi\left(q^{2}\right)$, is the cross-section reduction relative to that of the complete coherency condition [6], where

$$
\Gamma\left(q^{2}\right) \equiv \Gamma_{D A T A}\left(q^{2}\right)=(\varepsilon Z-N)^{2} \cdot \xi\left(q^{2}\right) .
$$

The functions $\Gamma_{N P}, \Gamma_{Q M}$, and $\Gamma_{D A T A}$ are complementary descriptions of the $\nu \mathrm{A}_{e l}$ interactions. The experimentally measurable cross-section reduction fraction $\left(\xi\right.$ in $\left.\Gamma_{D A T A}\right)$ is related to QM coherency ( $\alpha$ in $\Gamma_{Q M}$ ) and nuclear form factors via, respectively,

$$
\xi\left(q^{2}\right)=\alpha\left(q^{2}\right)+\left[1-\alpha\left(q^{2}\right)\right]\left[\frac{\left(\varepsilon^{2} Z+N\right)}{(\varepsilon Z-N)^{2}}\right]
$$


(a)

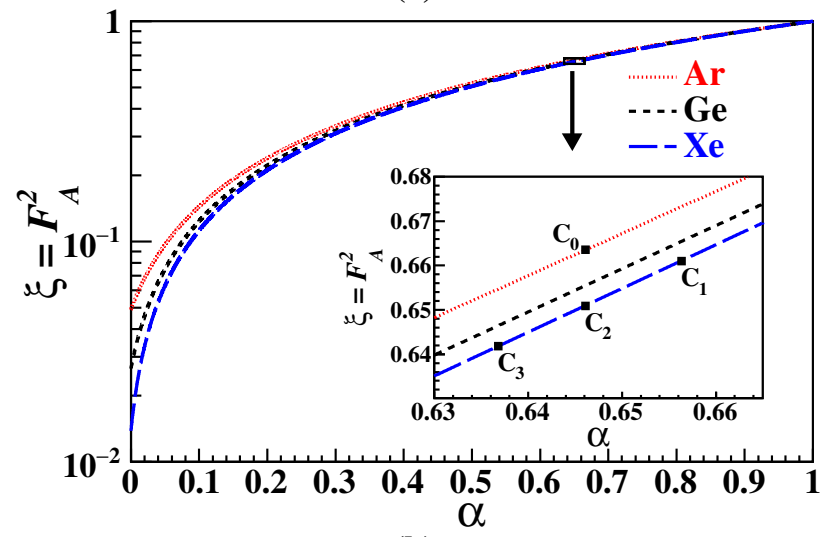

(b)

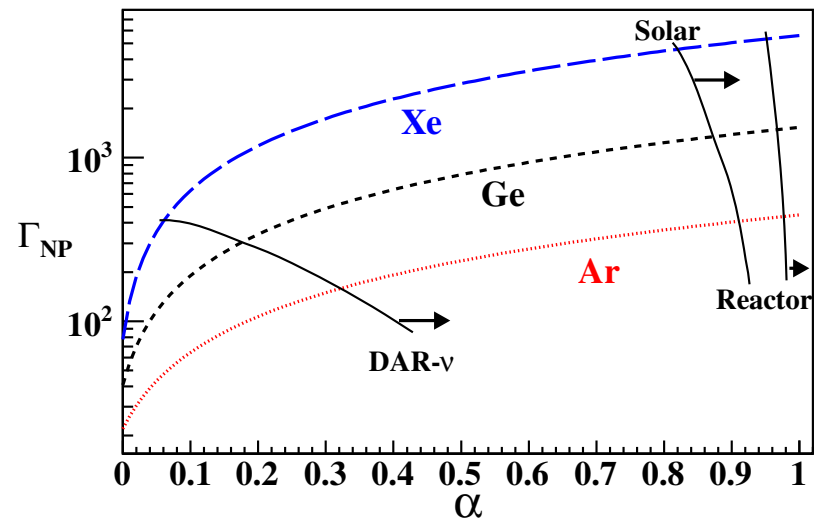

FIG. 1. The variations with $\alpha$ for (a) cross-section reduction fraction $\xi$ (equivalently $F_{\mathrm{A}}$ ) and (b) $\Gamma_{N P}$ for the three target nuclei, independent of the underlying nuclear physics. Superimposed in (b) are contours of maximum $q^{2}$ for reactor, solar, and DAR- $\pi$ neutrinos. The limiting values are $\xi=1$ and $\Gamma_{N P}=$ $(\varepsilon Z-N)^{2}$ at $\alpha=1$ and $\xi=\left(\varepsilon^{2} Z+N\right) /(\varepsilon Z-N)^{2}$ and $\Gamma_{N P}=$ $\left(\varepsilon^{2} Z+N\right)$ at $\alpha=0$. The configurations $\mathrm{C}_{0,1,2.3}$ in the inset of (a) illustrate the cases where $\alpha_{0}<\alpha_{1}, \alpha_{0}=\alpha_{2}$, and $\alpha_{0}>\alpha_{3}$ despite our having $F_{A}^{2}=\xi$ and $\xi_{0}>\xi_{1,2,3}$ in all cases.

and

$$
\xi\left(q^{2}\right)=\frac{\left[\varepsilon Z F_{Z}\left(q^{2}\right)-N F_{N}\left(q^{2}\right)\right]^{2}}{(\varepsilon Z-N)^{2}}
$$

while the two physics descriptions are connected by

$$
\begin{aligned}
& {\left[\varepsilon Z F_{Z}\left(q^{2}\right)-N F_{N}\left(q^{2}\right)\right]^{2}} \\
& \quad=(\varepsilon Z-N)^{2} \cdot \alpha\left(q^{2}\right)+\left(\varepsilon^{2} Z+N\right) \cdot\left[1-\alpha\left(q^{2}\right)\right] .
\end{aligned}
$$

The relations between $\xi$ and $\Gamma_{N P}$ with $\alpha$ for three representative nuclei are shown in Figs. 1(a) and 1(b), respectively. Contours of maximum $q^{2}$ for different neutrino sources are marked in Fig. 1(b). The behaviors of $\Gamma_{N P}, \alpha$, and $\xi$ at the limiting domains corresponding to the complete coherency and decoherency conditions are summarized in Table I. In particular, the relation
TABLE I. Summary of the three formulations which characterize the many-body physics in $\nu \mathrm{A}_{e l}$, and the values of the key parameters at the limiting domains where the scattering ampli-

\begin{tabular}{|c|c|c|}
\hline Conditions & $\begin{array}{l}\text { Complete } \\
\text { coherency }\end{array}$ & $\begin{array}{c}\text { Complete } \\
\text { decoherency }\end{array}$ \\
\hline$q^{2}$ & $\rightarrow 0$ & $\begin{array}{c}\gtrsim\left[\frac{\pi}{R}\right]^{2} \\
\text { with } A \text { dependence }\end{array}$ \\
\hline \multicolumn{3}{|c|}{ (I) $\Gamma_{N P}\left(q^{2}\right)=\left[\varepsilon Z F_{Z}\left(q^{2}\right)-N F_{N}\left(q^{2}\right)\right]^{2}$} \\
\hline$F_{Z}\left(q^{2}\right)$ & 1 & $\cdots$ \\
\hline$F_{N}\left(q^{2}\right)$ & 1 & $\cdots$ \\
\hline$\Gamma_{N P}\left(q^{2}\right)$ & $(\varepsilon Z-N)^{2}$ & $\left(\varepsilon^{2} Z+N\right)$ \\
\hline \multicolumn{3}{|c|}{ (II) $\Gamma_{Q M}\left(q^{2}\right)=(\varepsilon Z-N)^{2} \alpha\left(q^{2}\right)+\left(\varepsilon^{2} Z+N\right)\left[1-\alpha\left(q^{2}\right)\right]$} \\
\hline$\phi\left(q^{2}\right)$ & 0 & $\pi / 2$ \\
\hline$\alpha\left(q^{2}\right)$ & 1 & 0 \\
\hline \multicolumn{3}{|c|}{ (III) $\Gamma_{D A T A}\left(q^{2}\right)=(\varepsilon Z-N)^{2} \xi\left(q^{2}\right)$} \\
\hline$\xi\left(q^{2}\right)$ & 1 & {$\left[\frac{\left(\varepsilon^{2} Z+N\right)}{(\varepsilon Z-N)^{2}}\right]$} \\
\hline$\left[\frac{d \sigma}{d q^{2}}\right]\left(q^{2}\right)$ & $\propto(\varepsilon Z-N)^{2}$ & $\propto\left(\varepsilon^{2} Z+N\right)$ \\
\hline
\end{tabular}
tudes are either completely in phase ("coherency") or decoupled ("decoherency").

$\Gamma_{N P}=\left(\varepsilon^{2} Z+N\right)$ for completely decoherent $\nu \mathrm{A}_{e l}$ interactions is a result that emerges by relating $\Gamma_{N P}$ and $\Gamma_{Q M}$ in Eq. (8) and could not be derived by considerations of the nuclear form factor of Eq. (3) alone.

\section{PROJECTED EXPERIMENTAL RANGES}

The functions $\Gamma_{N P}, \Gamma_{Q M}$, and $\Gamma_{D A T A}$ can be directly measured from $\nu \mathrm{A}_{e l}$ data without input from the underlying physics. Prior to actual measurements, specific formulations of the nuclear form factors have to be adopted for phenomenological studies and to establish the typical ranges to guide the choices of experimental parameters. To serve these purposes, the frequently adopted approach is to ensure that the nuclear form factors for protons and neutrons are identical: $F_{N}\left(q^{2}\right)=F_{Z}\left(q^{2}\right) \equiv F_{\mathrm{A}}\left(q^{2}\right)$, and to use the effective "Helm form factor" description of Ref. [20]:

$$
F_{\mathrm{A}}\left(q^{2}\right)=\left[\frac{3}{q R_{0}}\right] j_{1}\left(q R_{0}\right) \exp \left[-\frac{1}{2} q^{2} s^{2}\right],
$$

where $j_{1}(x)=\left[\left(\sin x / x^{2}\right)-(\cos x / x)\right]$ is the first-order spherical Bessel function. The nuclear dependence appears through $R_{0}^{2}=R^{2}-5 s^{2}$, where $s=0.5 \mathrm{fm}$ is the surface thickness of the nuclei. In this formulation, the squaredform factor is equivalent to the cross-section reduction fraction: $\left[F_{\mathrm{A}}\left(q^{2}\right)\right]^{2}=\xi\left(q^{2}\right)$. 

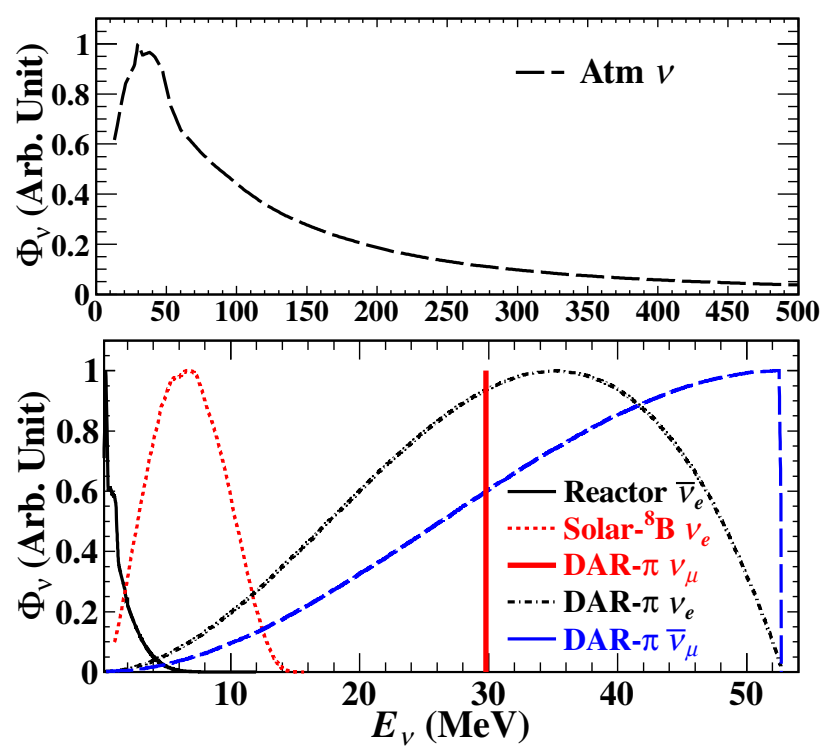

FIG. 2. Neutrino spectra $\left(\Phi_{\nu}\right)$ from (top panel) atmospheric as well as (bottom panel) reactor $\bar{\nu}_{e}$, solar ${ }^{8} \mathrm{~B} \nu_{e}$, and DAR- $\pi\left(\nu_{\mu}, \nu_{e}\right.$, $\bar{\nu}_{\mu}$ ) neutrinos adopted from Ref. [15,21] and normalized by their maxima.

Typical spectra $[15,21]$ of reactor, solar, and atmospheric neutrinos, as well as those due to DAR- $\pi$, are used in this study. These are depicted in Fig. 2.

The measurable total cross section is given by convoluting Eq. (2) with the neutrino spectrum $\Phi_{\nu}\left(E_{\nu}\right)$ and integrating over $E_{\nu}$ and $q^{2} \in\left[q_{\min }^{2}, q_{\max }^{2}\right]$, from which the mean reduction fraction $\langle\xi\rangle$ and the mean coherency factor $\langle\alpha\rangle$ can be derived [19].

The $\nu \mathrm{A}_{e l}$ processes on several nuclei of experimental interest and at different mass ranges are studied here: (Ar; $\mathrm{Ge} ; \mathrm{Xe})$ with $Z=(18 ; 32 ; 54)$. The target that provides the first $\nu \mathrm{A}_{e l}$ measurements [15] - CsI, with $Z=55$ and 53, respectively - can be approximated as Xe in this discussion.

The variations of $\alpha$ and $\xi\left(=F_{\mathrm{A}}^{2}\right)$ with $q^{2}$ of the four neutrino sources, with three selected nuclei ( $\mathrm{Ar} ; \mathrm{Ge} ; \mathrm{Xe})$, are depicted in Fig. 3. The $q^{2}$ dependence is universal for the different neutrino sources, although their $q_{\max }^{2}$ values are distinct due to their varying maxima $E_{\nu}$. These spectra end points for reactor, solar, and DAR- $\pi$ neutrinos are well defined, and their corresponding ranges in $\alpha$ and $q^{2}$ are depicted in Figs. 1(b) and 3, respectively.

A summary plot on the variations of $\langle\alpha\rangle$ with the neutrino sources and target nuclei is illustrated in Fig. 4, in which the ranges in $E_{\nu}$ are defined by the full width at half maximum (FWHM) of $\left[\Phi_{\nu} \cdot \sigma_{\nu \mathrm{A}_{e l}}\right]$. For completeness, the differential and integral event rates due to the four neutrino sources in measurable nuclear recoil energy $T$, together with their corresponding $\alpha$ and $\langle\alpha\rangle$ values, are discussed and presented in the Appendix.

It can be seen that coherency is mostly complete $(\alpha>95 \%)$ for $\nu \mathrm{A}_{e l}$ with reactor and solar neutrinos,

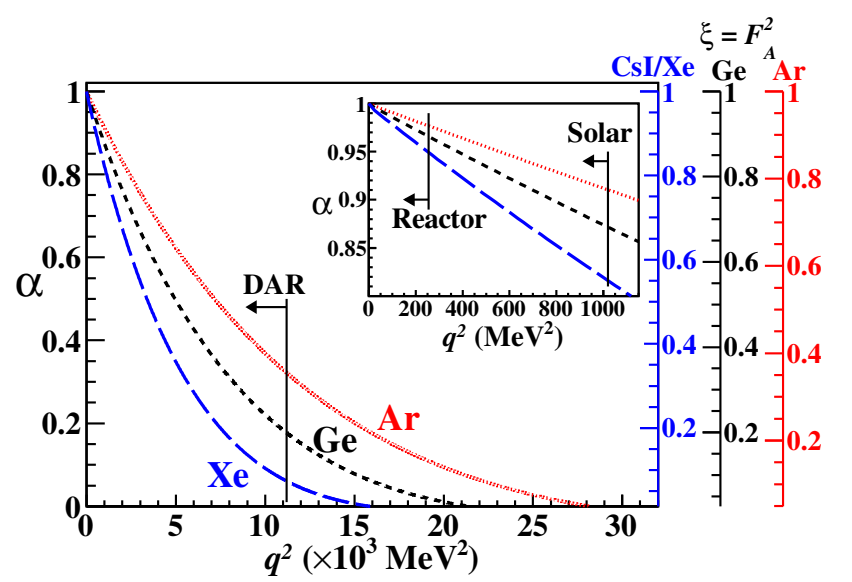

FIG. 3. The variation of $\alpha$ and $\xi\left(=F_{\mathrm{A}}^{2}\right)$ as a function of $q^{2}$ of $\nu \mathrm{A}_{e l}$ on the three selected nuclei. Different neutrino sources share the same contour for the same target in $q^{2}$ space, but with different ranges. The end points for reactor, solar, and DAR- $\pi$ neutrinos are marked.

whereas coherency is only partial for DAR- $\pi$ and weak for atmospheric neutrinos. Accordingly, studies of $\nu \mathrm{A}_{e l}$ with different neutrino sources provide complementary information and cover the transitions from completely coherent to decoherent states.

\section{MEASUREMENTS FROM CURRENT DATA}

The COHERENT CsI $(\mathrm{Na})$ and Ar experiments at the DAR- $\pi$ beam with the Spallation Neutron Source facility at the Oak Ridge National Laboratory have provided positive measurements on $\nu \mathrm{A}_{e l}$.

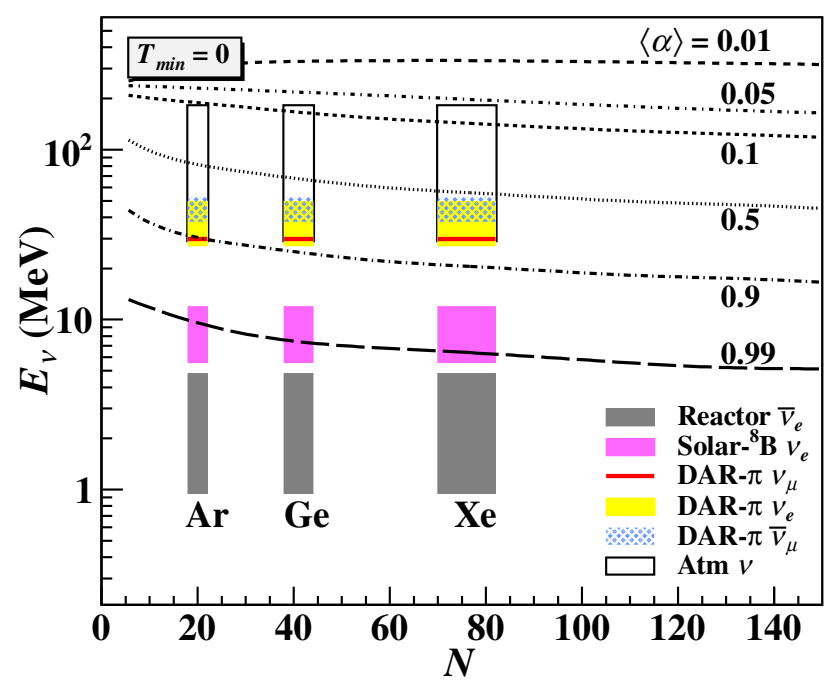

FIG. 4. The contours of the mean degree of coherency $\langle\alpha\rangle$ on the $\left(N, E_{\nu}\right)$ plane at $T_{\min }=0$, with bands of neutrino sources and target nuclei superimposed. The ranges in $E_{\nu}$ correspond to FWHM in $\left[\Phi_{\nu} \cdot \sigma_{\nu \mathrm{A}_{e l}}\right]$. 


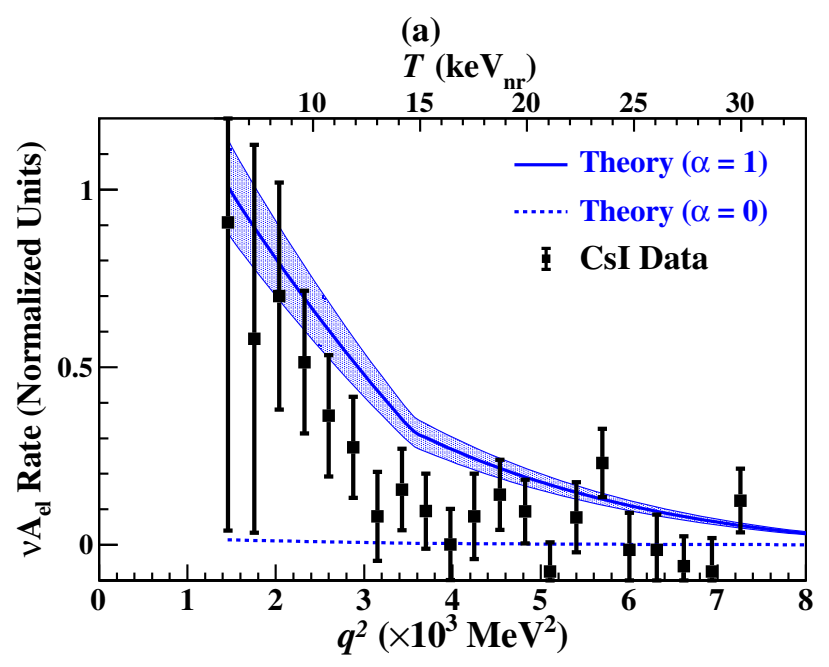

(b)

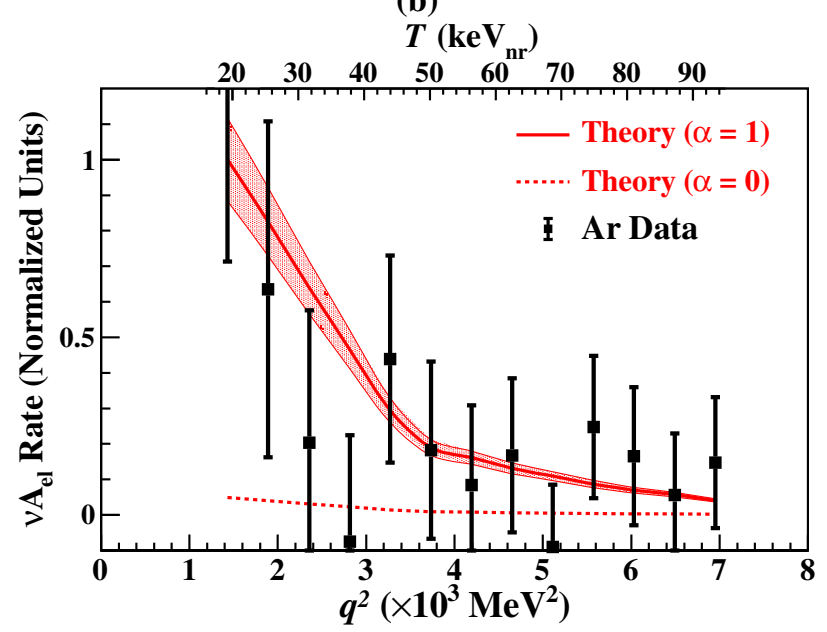

FIG. 5. Efficiency-corrected differential event rates with their statistical uncertainties derived from the COHERENT (a) CsI (Na) $[15,22]$ and (b) Ar [16] data. Superimposed are the predicted theory bands at the complete coherency $(\alpha=1)$ condition, where $\Gamma\left(q^{2}\right)=(\epsilon Z-N)^{2}$ is set in Eq. (2). Systematic uncertainties are represented by the width of the bands. Their ratios give rise to the cross-section reduction ratios $\xi$, from which $\alpha\left(q^{2}\right)$ is derived via Eq. (6). The allowed intervals and $p$ values follow from standard Gaussian statistics [24]. The maxima of the theory bands within the analyzed ranges are normalized to unity. The complete decoherency conditions $(\alpha=0)$ are denoted by dotted lines.

While the first-generation "discovery" measurements cannot be expected to provide severe constraints on $\alpha\left(q^{2}\right)$, it is instructive to go through the data analysis to establish the ranges of the effects and to check consistency.

The published event rates and statistical uncertainties from the COHERENT CsI(Na) [15] and Ar [16] data were adopted as input in this analysis. What were measured per event are the numbers of photoelectrons. To convert these to nuclear recoil energy for physics interpretation, knowledge of the quenching factor $(\mathrm{QF})$ is necessary. The $\mathrm{Ar}$ results of Ref. [16] already have QF incorporated. The uncertainties of the QF model used to derive the first results of CsI(Na) in Ref. [15] were at $\sim 25 \%$. For this analysis, a subsequent improved QF measurement by the same collaboration [22] was adopted in which an accuracy of $\sim 3.6 \%$ was stated. We note that a previous independent $\mathrm{QF}$ measurement [23] provided results consistent with this one at an $\sim 14 \%$ level.

Folding in published signal efficiencies, the efficiencycorrected event rates in different $q^{2}$ bins were derived and are depicted in Figs. 5(a) and 5(b) for CsI and Ar, respectively. These were compared with the complete coherency conditions in which $\alpha=1(\phi=0)$, and equivalently $\Gamma\left(q^{2}\right)=(\epsilon Z-N)^{2}$, is set in Eq. (2). Expected spectra for the completely decoherent cases are also displayed.

The systematic uncertainties of this analysis were taken from the published estimates discarding the component due to nuclear form factors. This corresponds to $11.7 \%$ [15,22] and $11.6 \%$ [16] for CsI and Ar, respectively. These are represented by the width of the complete coherency bands in Figs. 5(a) and 5(b). Systematic uncertainties are correlated in $q^{2}$ in general. In practice, statistical accuracies dominate the uncertainties at the current level of sensitivities, as shown by comparing the theory bandwidth with the data error bars in Figs. 5(a) and 5(b). Accordingly, the systematic errors are assumed to be uncorrelated in $q^{2}$. They are combined binwise in quadrature with the statistical errors to produce the total uncertainties. Under this error estimation scheme, the systematic effects contribute to $<4 \%$ and $<1.5 \%$ of the total uncertainties over all $q^{2}$ bins for CsI and Ar, respectively.

The cross-section reduction fractions $\xi\left(q^{2}\right)$ were evaluated, from which $\alpha\left(q^{2}\right)$ and their uncertainties were extracted using Eq. (6). The various measures which characterize allowed and excluded intervals at each $q^{2}$ bin were then derived with the standard statistics procedures [24], assuming Gaussian errors.

The allowed $1 \sigma$ ranges in $\alpha\left(q^{2}\right)$ are depicted as stripeshaded regions in Figs. 6(a) and 6(b) for CsI and Ar, respectively. These results are data driven without invoking nuclear physics input. Measurements and implications of every $q^{2}$ bin are independent of and uncorrelated with the others, and therefore the allowed intervals in adjacent bins are discontinuous.

The different $q^{2}$ bins become correlated when the nuclear form factors of Eq. (9) are adopted and imposed as theoretical expectations. The predicted parameter space from measurements with projected $10 \%$ uncertainty at the $\pm 1 \sigma$ level is superimposed as dark-shaded bands, showing the cases with CsI (equivalently, Xe) and Ar at DAR- $\pi$. The projected sensitivity for reactor $\nu$ on Ge is displayed in Fig. 6(c), showing that measurements with reactor $\nu \mathrm{A}_{e l}$ can probe the complete coherency regime. A binwise 10\% uncertainty corresponds to an appropriate sensitivity target for future experiments. It is the scale where systematic effects start to contribute, and the measurements can make 
(a)

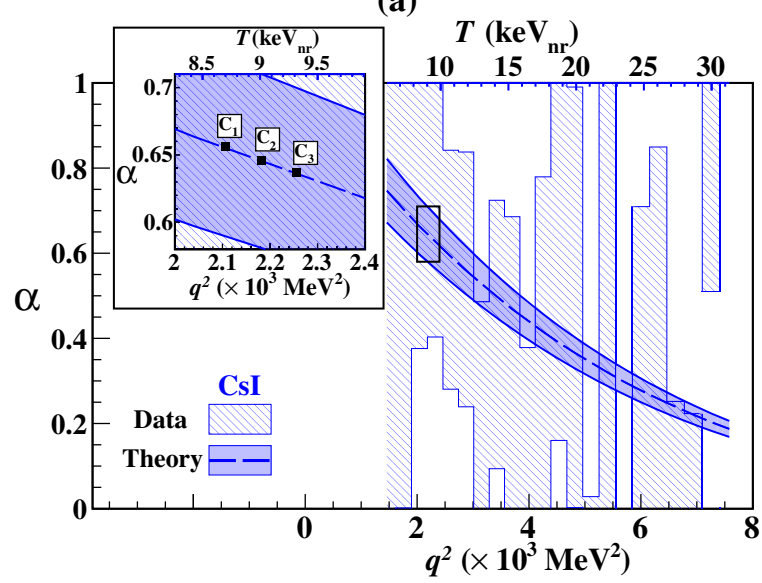

(b)

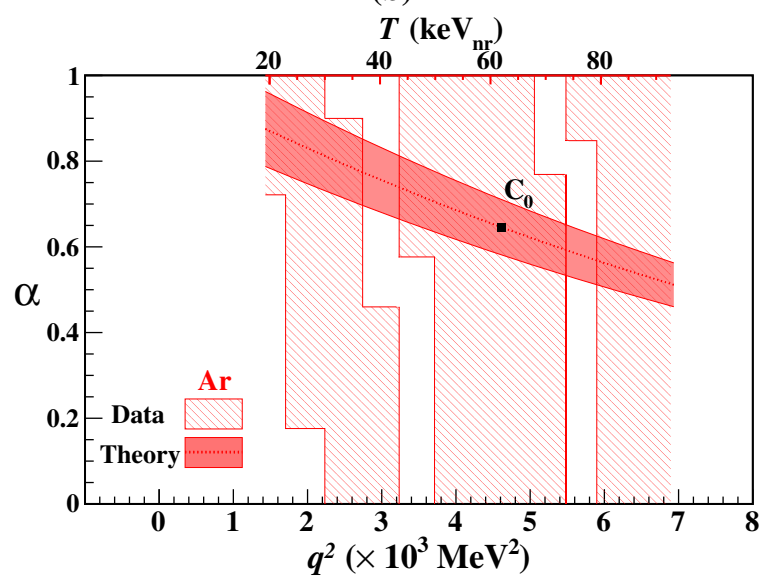

(c)

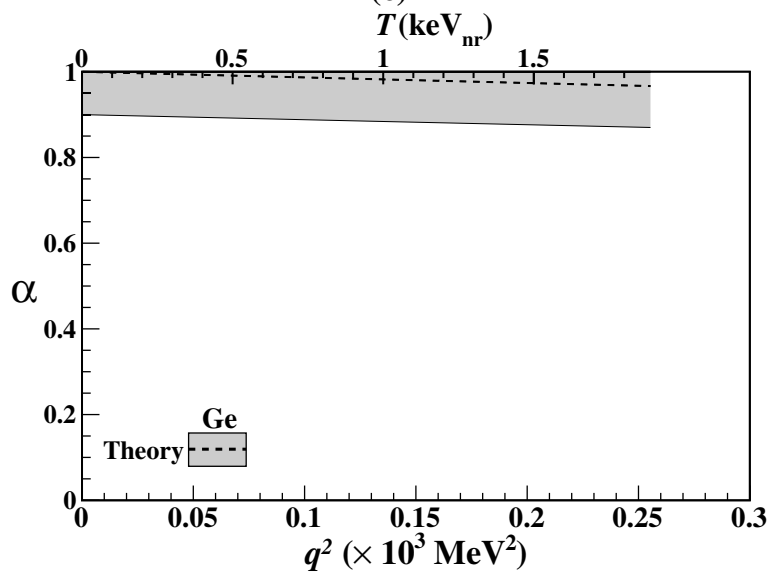

FIG. 6. Measurements on $\alpha$ from COHERENT (a) CsI [15,22] and (b) $\operatorname{Ar}$ [16] data with DAR- $\pi-\nu$. The stripe-shaded areas are the $1 \sigma$ allowed regions derived from the reduction in cross section relative to the complete coherency conditions independent of nuclear physics input. The dark-shaded regions are the theoretical expectations adopting the nuclear form factor formulation of Eq. (9) with a $\pm 1 \sigma$ uncertainty of $10 \%$. The $\alpha\left(q^{2}\right)$ values for different nuclei can be consistently compared. Labels $\mathrm{C}_{0,1,2,3}$ correspond to the configurations in Fig. 1(a), where $\alpha_{0}<\alpha_{1}$, $\alpha_{0}=\alpha_{2}$, and $\alpha_{0}>\alpha_{3}$ despite our having $\xi_{0}>\xi_{1,2,3}$ in all cases. (c) The sensitivity with the theoretical projections applied to reactor $\nu$ on Ge covering the complete $q^{2}$ range for nuclear recoils.

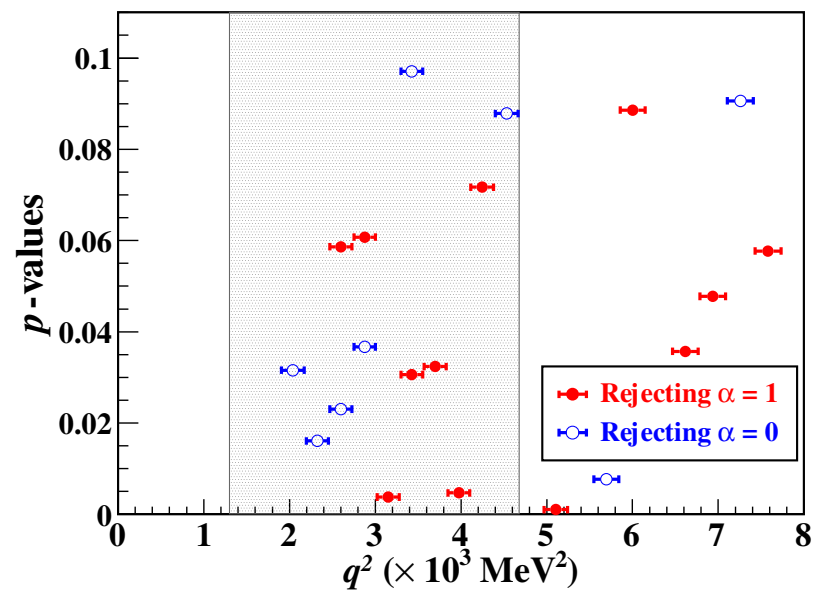

FIG. 7. The $p$-value significance to probe the specific cases corresponding to the complete coherency $(\alpha=1$ and $\phi=0$, in red) and decoherency ( $\alpha=0$ and $\phi=\pi / 2$, in blue) conditions from the COHERENT CsI data [15,22]. The shaded band corresponds to the stated region of interest in Ref. [15] where physics analysis was performed.

strong tests on the extreme cases of $\alpha=0(1)$, as well as comparisons with nuclear form factor predictions.

It can be seen that the current data are consistent with the predictions from Eq. (9). Future measurements with sufficient accuracies would probe the transitions in QM coherency according to

\section{CsI/Xe @ DAR- $\pi$ : \\ $\alpha \in[0.72,0.14] \quad$ for $T \in[6.6,36] \mathrm{keV}_{\mathrm{nr}}$}

Ar@DAR- $\pi$ :

$$
\alpha \in[0.88,0.51] \quad \text { for } T \in[19,93] \mathrm{keV}_{\mathrm{nr}}
$$

Ge @ Reactor $($ Projected $)$ :

$$
\alpha \in[1.00,0.96] \text { for } T \in[0,1.9] \mathrm{keV}_{\mathrm{nr}}
$$

following the $T$ ranges used in Fig. 6 .

The significance in terms of $p$ values [24] for testing the specific cases of $\alpha=1(0)$ [equivalently, $\phi=0(\pi / 2)$ ] with the CsI data are depicted in Fig. 7. In particular, the most stringent bounds within the stated region of interest in Ref. [15] in excluding complete QM coherency and decoherency at $90 \%$ confidence levels with specified $p$ values are, respectively,

$$
\begin{aligned}
& \alpha<0.57, \\
& \phi<0.61 \cdot(\pi / 2), \\
& p=0.004
\end{aligned}
$$

at $q^{2}=3.1 \times 10^{3} \mathrm{MeV}^{2}$ and 


$$
\begin{aligned}
& \alpha>0.30, \\
& \phi>0.80 \cdot(\pi / 2), \\
& p=0.016
\end{aligned}
$$

at $q^{2}=2.3 \times 10^{3} \mathrm{MeV}^{2}$. These results verify that both $\mathrm{QM}$ superpositions among the nucleonic scattering centers and nuclear many-body effects contribute to the $\nu \mathrm{A}_{e l}$ process.

These diverse ranges of $\alpha$ sensitivity indicate the complementarity of $\nu \mathrm{A}_{e l}$ measurements among reactor and DAR- $\pi$ neutrinos. Future measurements of solar $\nu \mathrm{A}_{e l}[14]$ with multiton detectors would probe a similar range of $\alpha$ as reactor neutrinos. Xenon detectors with a scale $\mathcal{O}(100)$ ton would be required to probe the weakly coherent region at $\alpha<0.2$ with atmospheric neutrinos.

\section{SUMMARY AND PROSPECTS}

Neutrino-nucleus elastic scattering-the interaction $\nu \mathrm{A}_{e l}$ in Eq. (1) -involves two distinct concepts: elastic kinematics and QM coherency. It provides a laboratory to study QM superpositions in electroweak interactions. The QM coherency aspect should be characterized by distributions with dependence on $A(Z, N)$ and $q^{2}$. Descriptions of coherency as a binary state or having both concepts bundled together may have the unintended consequences of missing the complexities of the process and suppressing the potential richness of its physics content. We formulated a quantitative and universal parametrization of the QM coherency [6] to facilitate studies of QM effects in $\nu \mathrm{A}_{e l}$, under which the fundamental parameter is the experimentally accessible misalignment phase angle ( $\phi$, equivalently given as $\alpha \equiv \cos \phi$ ) between nonidentical nucleonic scattering centers.

Current positive measurements on $\nu \mathrm{A}_{e l}$ provide only weak constraints to $\alpha\left(q^{2}\right)$ and, equivalently, misalignment phase angle $\phi\left(q^{2}\right)$. Data with $\mathcal{O}(10 \%)$ accuracy would allow the studies of transitions in QM coherency over a wide range in $\alpha \in[0,1]$.

The $\nu \mathrm{A}_{e l}$ process described in terms of QM effects $\left(\Gamma_{Q M}\right)$ is complementary to the conventional descriptions with nuclear form factors $\left(\Gamma_{N P}\right)$ based on the many-body physics in the nucleon-nucleus interplay. Both formulations are related to the directly measurable cross-section reduction fraction $(\xi)$, and themselves, via Eqs. (6)-(8).

The $\alpha$ parameter quantifies QM coherency in $\nu \mathrm{A}_{e l}$ and adds preciseness to the qualitative discussions. While the derivation of the $\alpha$ values depends on $A(Z, N)$ and $q^{2}$ in similar footing as those for $\Gamma_{N P}$, the interpretation of measurements with $\alpha$ in terms of degrees of QM coherency or scattering phase-angle alignment is universal among different configurations with varying nuclei and $q^{2}$. This feature allows the quantitative characterization of the configurations and is not available with the $\xi$ or $\Gamma_{N P}$ frameworks. Referring to an example illustrated in Fig. 1(a), the four cases $\mathrm{C}_{0,1,2,3}$ are all within the
DAR- $\pi$ measurable kinematics domain. It can be inferred that configuration $\mathrm{C}_{0}$ with Ar has the same QM coherency as $\mathrm{C}_{2}$ in CsI $\left(\alpha_{0}=\alpha_{2}\right)$ while having lower and higher levels of coherency than $\mathrm{C}_{1}\left(\alpha_{0}<\alpha_{1}\right)$ and $\mathrm{C}_{3}\left(\alpha_{0}>\alpha_{3}\right)$, respectively, despite the fact that the nuclear targets and interaction kinematics $\left(q^{2}\right.$ and $\left.T\right)$ are different and the cross-section reduction $\xi_{0}>\xi_{1,2,3}$ apply in all cases.

New measurements on $\nu \mathrm{A}_{e l}$ from a variety of neutrino sources and nuclear targets can be expected. To facilitate comprehensive bookkeeping of the expanding array of data from diverse configurations, it would be beneficial to include the $\alpha$ parameter as a qualifier on QM coherency to each measurement, in a similar spirit as adopting $q^{2}$ to qualify the interaction kinematics.

The quantitative description of QM superpositions with the $\alpha$ parameter among the scattering amplitudes between individual nucleons may serve as natural entry points to some BSM studies, such as those where both coherent and decoherent channels would contribute to $\nu \mathrm{A}_{e l}$ [25]. The experimentally measurable relation between $\Gamma_{N P}$ and $\alpha$ in Fig. 1(b) describes the transitions in QM coherency in terms of the evolution from nuclear to nucleon effects in a $\nu \mathrm{A}_{e l}$ interaction. Understanding and applications of these are possible topics of future research but are beyond the scope of this work.

\section{ACKNOWLEDGMENTS}

This work is supported by Academia Sinica Principal Investigator Award No. AS-IA-106-M02, Contracts No. 106-2923-M-001-006-MY5, No. 107-2119-M-001028-MY3, and No. 109-2112-M-259-001 from the Ministry of Science and Technology, Taiwan, and Grant No. 2017-ECP2 from the National Center of Theoretical Sciences, Taiwan. We are grateful to the reviewer for recently bringing to our attention the new quenching factor measurements given in Ref. [22].

\section{APPENDIX: MEASURABLE EVENT RATES}

The differential cross section of Eq. (2) on $q^{2}$ can be translated into one as a function of the measurable nuclear recoi energy $T$ by

$$
\left[\frac{d \sigma}{d T}\right]_{\nu \mathrm{A}_{e l}}=2 M\left[\frac{d \sigma}{d q^{2}}\right]_{\nu \mathrm{A}_{e l}} .
$$

The differential spectra $(d R / d T)$ convoluted with the neutrino spectrum $\Phi_{\nu}\left(E_{\nu}\right)$ is given by

$$
\left(\frac{d R}{d T}\right)_{\nu \mathrm{A}_{e l}}=2 M \int\left[\left(\frac{d \sigma}{d q^{2}}\right)_{\nu \mathrm{A}_{e l}}\left(T, E_{\nu}\right)\right] \Phi_{\nu}\left(E_{\nu}\right) d E_{\nu} .
$$

Integration over $T \in\left[T_{\min }, T_{\max }\right]$ gives the total event rates. 
(a)

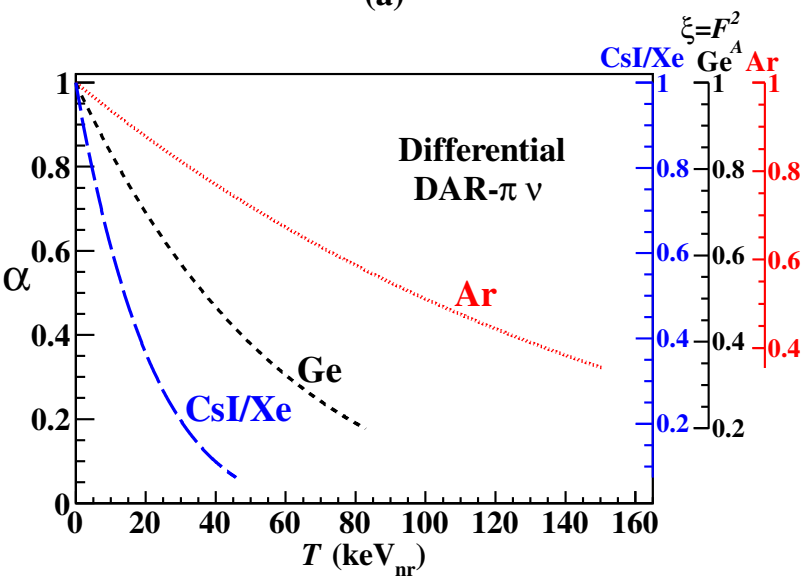

(c)

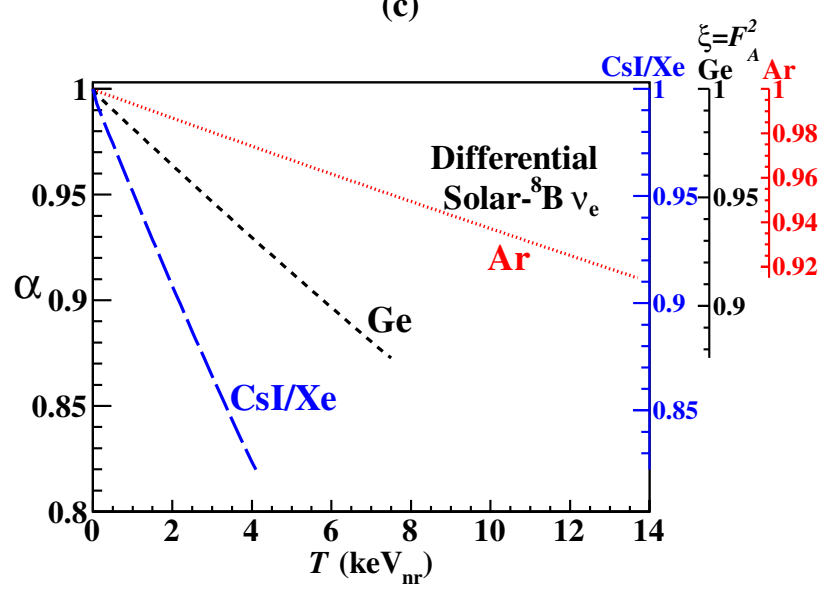

(b)

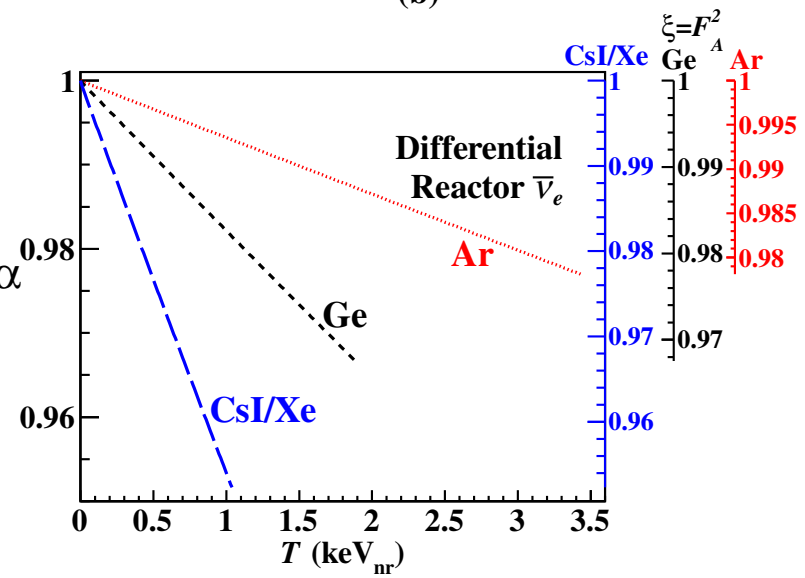

(d)

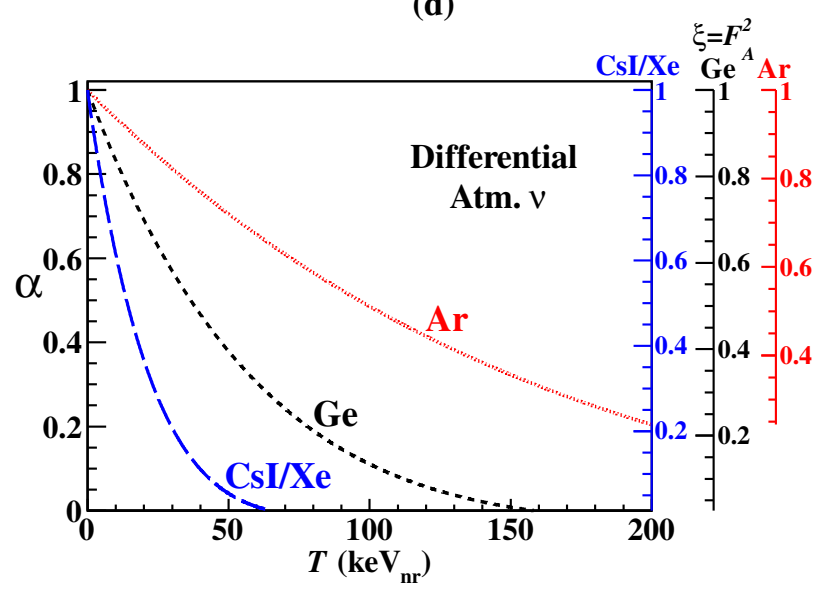

FIG. 8. The variations of $\alpha$ and $\xi\left(=F_{\mathrm{A}}^{2}\right)$ with $T$ in $\nu \mathrm{A}_{e l}$ on the three selected nuclei for (a) DAR- $\pi$, (b) reactor, (c) solar, and (d) atmospheric neutrinos.

The universality of Fig. 3 no longer applies when $q^{2}$ is replaced by $T$. The variations of $\alpha, F_{\mathrm{A}}$, and $\xi$ with $T$ depend on $E_{\nu}$ distributions, and therefore also neutrino sources. The variations are depicted in Fig. 8.

The differential rates derived from the four sources and three targets are displayed in Fig. 9. The corresponding total rates are shown in Fig. 10, showing their variations with $T_{\min }$ and $\langle\alpha\rangle$. The values of $\langle\alpha\rangle$ and $\langle\xi\rangle$ as well as the total event rates at $T_{\min }=1(10) \mathrm{keV}_{\mathrm{nr}}$ for the various neutrino sources and target nuclei are summarized in Table II. Evaluation of these rates are based on standard solar and atmospheric spectra $[15,21]$. Reactor $\bar{\nu}_{e}$ flux is taken to be $10^{13} \mathrm{~cm}^{-2} \mathrm{~s}^{-1}$, while DAR- $\pi$ per-flavor neutrino flux is $3.4 \times$ $10^{14} \mathrm{~cm}^{-2} \mathrm{yr}^{-1}$ corresponding to $2 \times 10^{23}$ proton-on-target (POT) $/ \mathrm{yr}$ at $19.3 \mathrm{~m}$ from the target [15] There is no highenergy cutoff in $E_{\nu}$ for the atmospheric neutrino spectra. The differential and integral spectra of Figs. 9 and 10(d) are therefore evaluated for $\alpha \in[0.01,1.0]$, corresponding to $T<(361 ; 148 ; 60) \mathrm{keV}_{\mathrm{nr}}$ for $(\mathrm{Ar} ; \mathrm{Ge} ; \mathrm{Xe})$.
Typically, measurements of $\nu \mathrm{A}_{e l}$ with reactor and solar neutrinos require an $\mathcal{O}(1) \mathrm{keV}_{\mathrm{nr}}$ detector threshold giving expected rates of $\mathcal{O}(1) / \mathrm{kg}$ day and $\mathcal{O}(1) /$ ton yr, respectively. The corresponding event rates for DAR- $\pi$ and atmospheric neutrinos are $\mathcal{O}(10) / \mathrm{kg}$ yr and $\mathcal{O}(0.01) /$ ton yr, respectively, at a threshold of $\mathcal{O}(10) \mathrm{keV}_{\mathrm{nr}}$.

At the detection threshold of $1 \mathrm{keV}_{\mathrm{nr}}, 90 \%$ of the elastic scattering events between weakly interacting massive particles (WIMPs) and dark matter with a mass of $1 \mathrm{TeV}$ and an (Ar;Ge;Xe) target have recoil energy up to $(99 ; 74 ; 35) \mathrm{keV}_{\mathrm{nr}}$. These kinematics ranges correspond to $\alpha$ as low as $(0.49 ; 0.22 ; 0.14)$ for $\nu \mathrm{A}_{e l}$ scattering with atmospheric neutrinos, as indicated in Fig. 8d-which is far from the complete coherency regime. Accordingly, the description "the neutrino floor originates from coherent neutrinonucleus scattering" is not applicable to WIMPs at $\mathrm{TeV}$ or higher mass scales. 
(a)

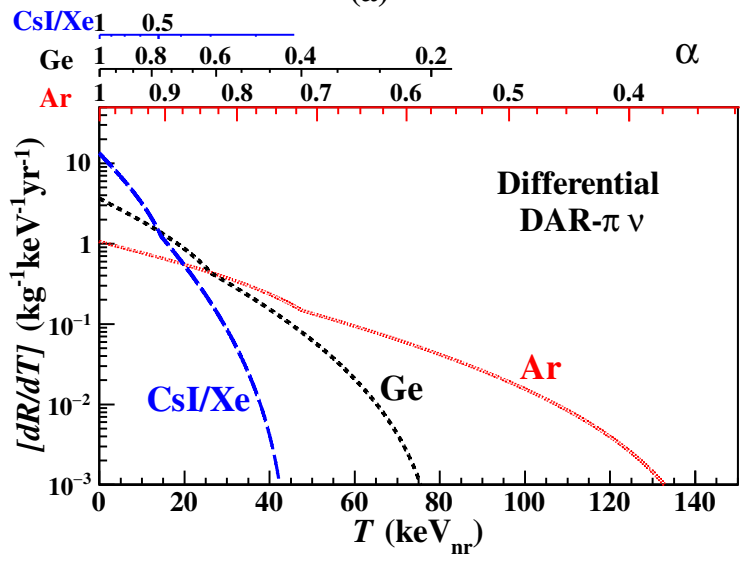

(c)

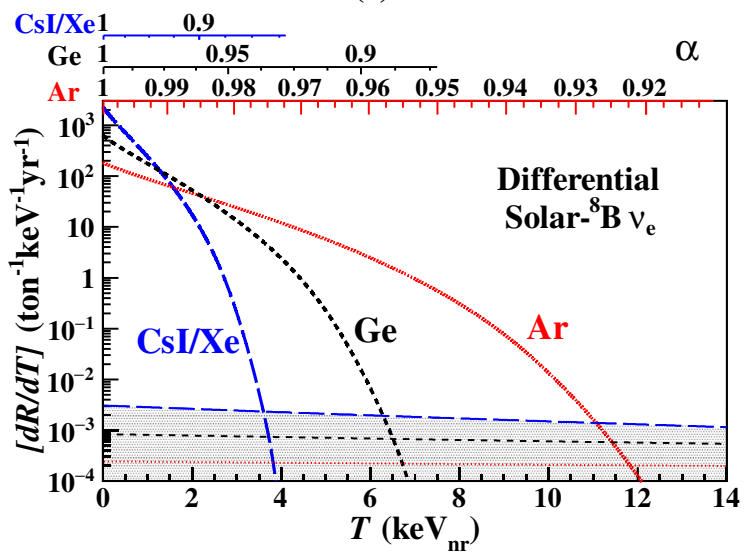

(b)

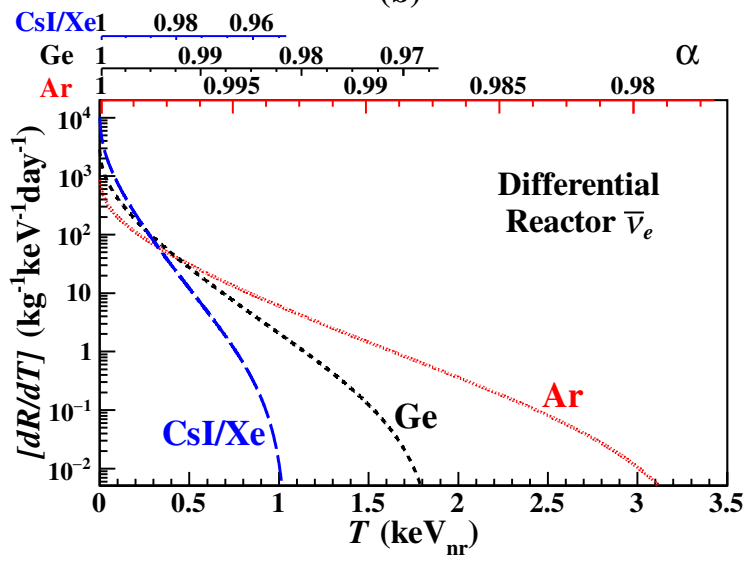

(d)

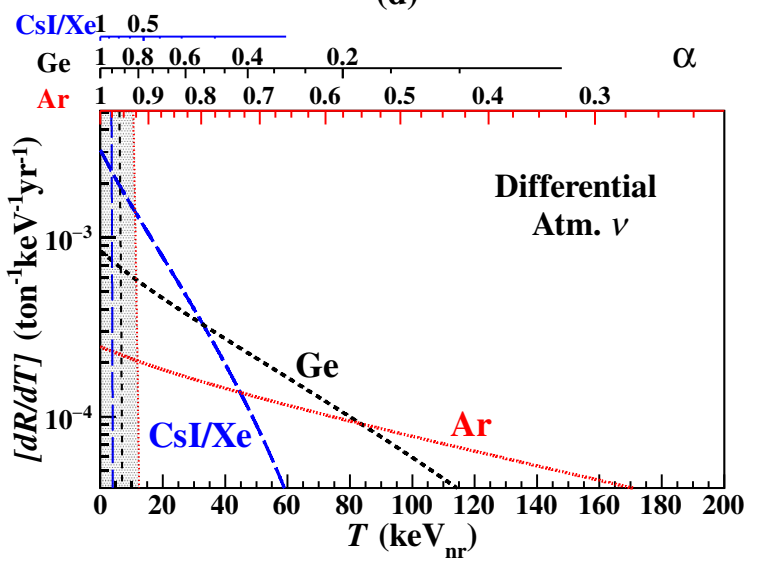

FIG. 9. Differential event rates $[d R / d T]$ of $\nu \mathrm{A}_{e l}$ on the three selected nuclei, and their correlations with $\alpha$ and with (a) DAR- $\pi$, (b) reactor, (c) solar, and (d) atmospheric neutrinos. Superimposed as shaded regions in (c) and (d) are the background rates due to atmospheric and solar neutrinos, respectively. 
(a)

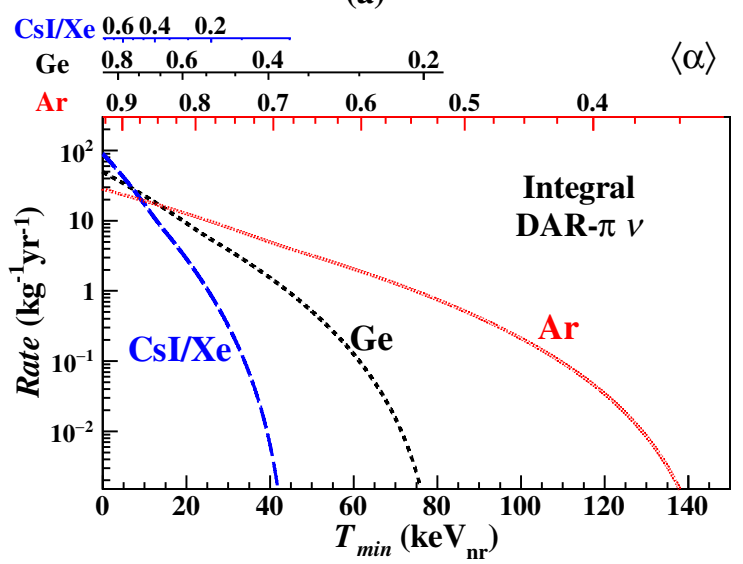

(c)

CsI/Xe $0.95 \quad 0.9 \quad 0.85$

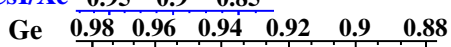

$\begin{array}{lllllllll}\text { Ar } & 0.99 & 0.98 & 0.97 & 0.96 & 0.95 & 0.94 & 0.93 & 0.92\end{array}$

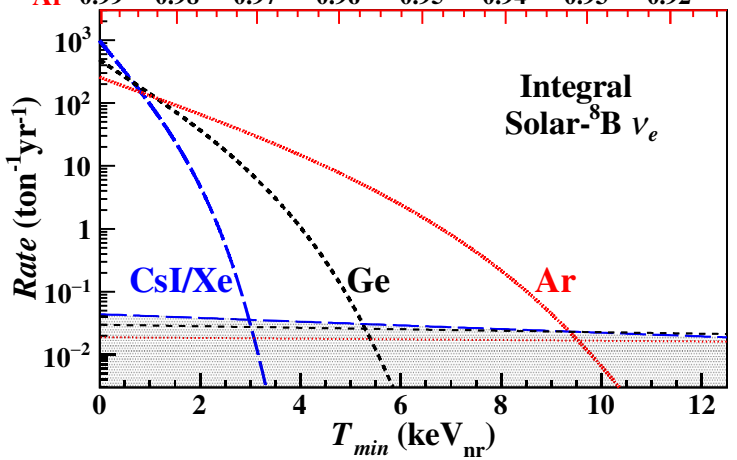

(b)

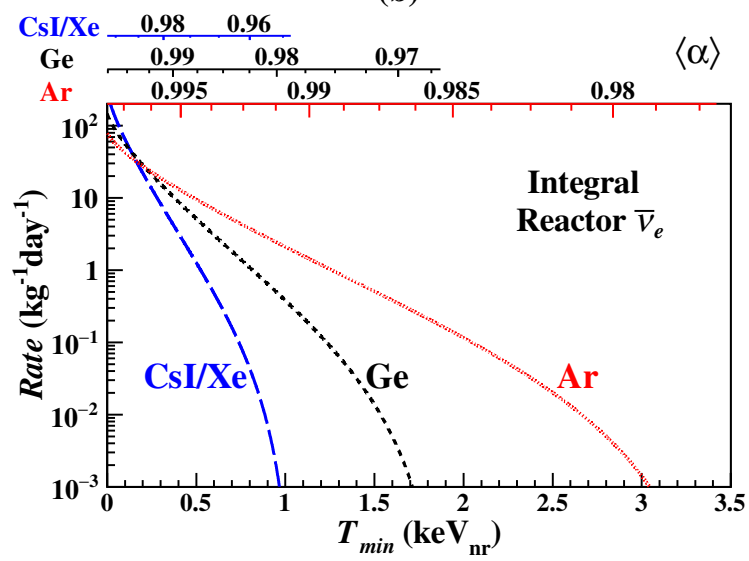

(d)

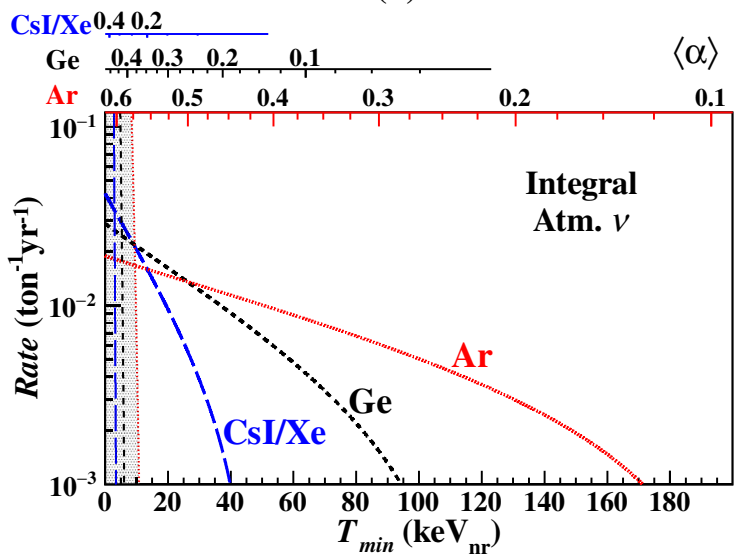

FIG. 10. Integral event rates of $\nu \mathrm{A}_{e l}$ on the three selected nuclei as functions of threshold $T_{\min }$, and their correlations with $\langle\alpha\rangle$ for (a) DAR- $\pi$, (b) reactor, (c) solar, and (d) atmospheric neutrinos. Superimposed as shaded regions in (c) and (d) are the background rates due to atmospheric and solar neutrinos, respectively.

TABLE II. Averaged $[\langle\alpha\rangle ;\langle\xi\rangle]$ and total event rates in $\mathrm{kg}^{-1}$ day $^{-1}$ for the target nuclei at a threshold of 1 and $10 \mathrm{keV}_{\mathrm{nr}}$ and for different $\nu$ sources. Reactor and DAR- $\pi$ neutrino fluxes are taken to be $10^{13} \mathrm{~cm}^{-2} \mathrm{~s}^{-1}$, while DAR- $\pi$ neutrino flux is $3.4 \times 10^{14} \mathrm{~cm}^{-2} \mathrm{yr}^{-1} /$ flavor at $19.3 \mathrm{~m}$ from the target at beam intensity $2 \times 10^{23} \mathrm{POT} \mathrm{yr}^{-1}$. Rates due to atmospheric neutrinos are from the integration of $q^{2}$ ranges corresponding to $\alpha \in[0.01,1.0]$.

\begin{tabular}{|c|c|c|c|c|}
\hline \multirow[b]{3}{*}{ Detector target } & \multicolumn{4}{|c|}{$\nu$ sources } \\
\hline & $\mathrm{DAR}-\pi$ & Reactor & Solar & Atmospheric \\
\hline & \multicolumn{2}{|c|}{$\begin{array}{c}{[\langle\alpha\rangle ;\langle\xi\rangle]} \\
\text { Total event rates }\left(\mathrm{kg}^{-1} \mathrm{yr}^{-1}\right)\end{array}$} & \multicolumn{2}{|c|}{$\begin{array}{c}{[\langle\alpha\rangle ;\langle\xi\rangle]} \\
\text { Total event rates }\left(\operatorname{ton}^{-1} \mathrm{yr}^{-1}\right)\end{array}$} \\
\hline & & Detect & $1 \mathrm{keV}_{\mathrm{nr}}$ & \\
\hline \multirow[t]{2}{*}{$\mathrm{Ar}$} & {$[0.92 ; 0.93]$} & {$[0.99 ; 0.99]$} & {$[0.98 ; 0.98]$} & {$[0.61 ; 0.63]$} \\
\hline & 27.2 & 766 & 130 & 0.019 \\
\hline \multirow[t]{2}{*}{$\mathrm{Ge}$} & {$[0.84 ; 0.84]$} & {$[0.98 ; 0.98]$} & {$[0.97 ; 0.97]$} & {$[0.46 ; 0.47]$} \\
\hline & 46.1 & 138 & 140 & 0.028 \\
\hline \multirow[t]{2}{*}{$\mathrm{Xe}$} & {$[0.72 ; 0.72]$} & {$[0.95 ; 0.95]$} & {$[0.94 ; 0.94]$} & {$[0.41 ; 0.42]$} \\
\hline & 77.8 & 0.07 & 95.7 & 0.039 \\
\hline & \multicolumn{4}{|c|}{ Detector threshold $=10 \mathrm{keV}_{\mathrm{nr}}$} \\
\hline \multirow[t]{2}{*}{ Ar } & {$[0.87 ; 0.87]$} & & & {$[0.57 ; 0.59]$} \\
\hline & 19.2 & & & 0.017 \\
\hline \multirow[t]{2}{*}{$\mathrm{Ge}$} & {$[0.72 ; 0.72]$} & & & {$[0.37 ; 0.39]$} \\
\hline & 22.6 & & & 0.022 \\
\hline \multirow[t]{2}{*}{$\mathrm{Xe}$} & {$[0.46 ; 0.47]$} & & & {$[0.24 ; 0.25]$} \\
\hline & 17.1 & & & 0.021 \\
\hline
\end{tabular}


[1] D. Z. Freedman, Phys. Rev. D 9, 1389 (1974); D. Z. Freedman, D. N. Schramm, and D. L. Tubbs, Annu. Rev. Nucl. Part. Sci. 27, 167 (1977).

[2] D. K. Papoulias and T. S. Kosmas, Adv. High Energy Phys. 2015, 763648 (2015).

[3] L. M. Krauss, Phys. Lett. B 269, 407 (1991); J. Barranco, O. G. Miranda, and T. I. Rashba, J. High Energy Phys. 12 (2005) 021; Phys. Rev. D 76, 073008 (2007); D. K. Papoulias et al., Phys. Rev. D 102, 113004 (2020).

[4] K. Scholberg, Phys. Rev. D 73, 033005 (2006).

[5] J. R. Wilson, Phys. Rev. Lett. 32, 849 (1974).

[6] S. Kerman, V. Sharma, M. Deniz, H. T. Wong, J.-W. Chen, H. B. Li, S. T. Lin, C.-P. Liu, and Q. Yue, Phys. Rev. D 93, 113006 (2016).

[7] K. Patton, J. Engel, G. C. McLaughlin, and N. Schunck, Phys. Rev. C 86, 024612 (2012); E. Ciuffoli, J. Evslin, Q. Fu, and J. Tang, Phys. Rev. D 97, 113003 (2018); M. Cadeddu, C. Giunti, Y. F. Li, and Y. Y. Zhang, Phys. Rev. Lett. 120, 072501 (2018); D. A. Sierra, J. Liao, and D. Marfatia, J. High Energy Phys. 06 (2019) 141; C. G. Payne, Phys. Rev. C 100, 061304(R) (2019); P. Coloma, I. Esteban, M. C. Gonzalez-Garcia, and J. Menéndez, J. High Energy Phys. 08 (2020) 030.

[8] C. J. Horowitz, K. J. Coakley, and D. N. McKinsey, Phys. Rev. D 68, 023005 (2003).

[9] J. Learned, Nucl. Phys. B, Proc. Suppl. 143, 152 (2005).

[10] J. Monroe and P. Fisher, Phys. Rev. D 76, 033007 (2007); A. Gütlein, C. Ciemniak, F. von Feilitzsch, N. Haag, M. Hofmann, C. Isaila, T. Lachenmaier, J.-C. Lanfranchi, L. Oberauer, and S. Pfister, Astropart. Phys. 34, 90 (2010); J. Billard, E. Figueroa-Feliciano, and L. Strigari, Phys. Rev. D 89, 023524 (2014).

[11] M. Drees and G. Gerbier, Phys. Rev. D 98, 030001:396 (2018), and references therein.

[12] H. T. Wong, H. B. Li, J. Li, Q. Yue, and Z. Y. Zhou, J. Phys. Conf. Ser. 39, 266 (2006); V. Belov et al., J. Instrum. 10, P12011 (2015); G. F. Moroni, J. Estrada, E. E. Paolini, G. Cancelo, J. Tiffenberg, and J. Molina, Phys. Rev. D 91, 072001 (2015); A. K. Soma et al., Nucl. Instrum. Methods
Phys. Res., Sect. A 836, 67 (2016); G. Agnolet et al., Nucl. Instrum. Methods Phys. Res., Sect. A 853, 53 (2017); C. Buck et al., J. Phys. Conf. Ser. 1324, 012094 (2020); J. Roth et al., J. Low Temp. Phys. 199, 433 (2020).

[13] F. T. Avignone and Yu. V. Efremenko, J. Phys. G 29, 2615 (2003); D. Akimov et al., arXiv:1509.08702, and references therein for current experimental projects.

[14] L. E. Strigari et al., New J. Phys. 11, 105011 (2009); L. Baudis, A. Ferella, A. Kish, A. Manalaysay, T. Marrodán Undagoitia, and M. Schumann, J. Cosmol. Astropart. Phys. 01 (2014) 044.

[15] J. I. Collar, N. E. Fields, M. Hai, T. W. Hossbach, J. L. Orrell, C. T. Overman, G. Perumpilly, and B. Scholz, Nucl. Instrum. Methods Phys. Res., Sect. A 773, 56 (2015); D. Akimov et al., Science 357, 1123 (2017), and Supplemental Material.

[16] D. Akimov et al., Phys. Rev. D 100, 115020 (2019); D. Akimov et al., Phys. Rev. Lett. 126, 012002 (2021), and Supplemental Material.

[17] I. Angeli and K. P. Marinova, At. Data Nucl. Data Tables 99, 69 (2013); J. E. Amaro et al., arXiv:1912.1061.

[18] T. W. Donnelly, J. Dubach, and I. Sick, Nucl. Phys. A503, 589 (1989).

[19] The $\alpha$ and $\xi$ results of Ref. [6] are mean values equivalent to $\langle\alpha\rangle$ and $\langle\xi\rangle$, respectively, in this article.

[20] J. Engel, Phys. Lett. B 264, 114 (1991).

[21] J. N. Bahcall, and M. H. Pinsonneault, Phys. Rev. Lett. 92, 121301 (2004); G. Battistoni, A. Ferrari, T. Montaruli, and P. R. Sala, Astropart. Phys. 23, 526 (2005); H. T. Wong et al., Phys. Rev. D 75, 012001 (2007).

[22] A. Konovalov, in Magnificent CEvNS, edited by P. Barbeau et al. (2020), https://indico.cern.ch/event/ 943069/contributions/4066385/.

[23] J. I. Collar, A. R. L. Kavner, and C. M. Lewis, Phys. Rev. D 100, 033003 (2019).

[24] G. Cowan, Phys. Rev. D 98, 030001:527 (2018).

[25] M. Hoferichter, J. Menendez, and A. Schwenk, Phys. Rev. D 102, 074018 (2020). 